\title{
Valuación de opciones sobre índices de la temperatura de la Ciudad de México
}

\author{
Option's pricing on Mexico City temperature indices \\ José Antonio Climent Hernández*, Domingo Rodríguez Benavides \\ Universidad Autónoma Metropolitana, México
}

Recibido el 25 de septiembre de 2019; aceptado el 12 de octubre de 2020

Disponible en Internet el: 21 de octubre de 2020

\section{Resumen}

En este trabajo son presentadas innovaciones para valuar opciones sobre índices de temperatura suponiendo que una organización necesita una cobertura, el modelo propuesto es obtenido aplicando una ecuación diferencial estocástica con procesos $\alpha$-estables, analizando el comportamiento de las temperaturas del observatorio de Tacubaya en el periodo de 01/01/1958 al 31/12/2018, estimando los estadísticos descriptivos, un modelo determinista de reversión a la media significativo y proponiendo un modelo estocástico de reversión a la media con procesos $\alpha$-estables, realizando un análisis mensual de las temperaturas, estimando los parámetros $\alpha$-estables y justificando la pertinencia de las distribuciones $\alpha$-estables con pruebas de bondad de ajuste, estimando el parámetro de reversión a la media para valuar opciones europeas sobre índices de temperatura con procesos $\alpha$-estables y cuantificar la cobertura, concluyendo que las opciones $\alpha$-estables son significativas, minimizan los costos por consumo energético y presentan un comportamiento similar a las opciones gaussianas pero con un costo menor.

Código JEL: C46, G13, G32

Palabras clave: Procesos $\alpha$-estables; Derivados climáticos; Ingeniería financiera

\footnotetext{
*Autor para correspondencia

Correo electrónico:jach@azc.uam.mx (J.A. Climent Hernández).

La revisión por pares es responsabilidad de la Universidad Nacional Autónoma de México. 


\begin{abstract}
In this work are proposed the innovations to option's pricing on temperature indices assuming that a company needs a hedge, the proposed model is obtained by applying a stochastic differential equation with $\alpha$-stable processes, analyzing the behavior of the Tacubaya central observatory temperatures during the period from $01 / 01 / 1958$ to $12 / 31 / 2018$, estimating descriptive statistics, a significant deterministic mean reversion model and proposing a stochastic mean reversion model with $\alpha$-stable processes, carrying out a monthly analysis of temperatures, estimating the $\alpha$-stable parameters and justifying the relevance of the $\alpha$-stable distributions with goodness of fit tests, estimating the mean reversion parameter to European call option's pricing on temperature indices with $\alpha$-stable processes and quantify the hedge, concluding that the $\alpha$-stable options are significant, minimize costs for energy consumption and have a similar behavior to the Gaussian options but with a lower cost.
\end{abstract}

JEL Code: C46, G13, G32

Keywords: The $\alpha$-stable processes; Weather derivatives; Financial engineering

\title{
Introducción
}

Suponiendo que una empresa de la delegación Miguel Hidalgo de la ciudad de México necesita una cobertura por el costo de consumo energético para calefacción o refrigeración replicando la posición larga en el valor actual que la empresa está dispuesta a pagar por el índice de temperatura y la posición larga en una opción europea de compra sobre el índice de temperatura, es decir, una estrategia de cobertura con respecto al costo de energía que indica la cantidad que la empresa ahorra y que minimiza el costo de consumo energético derivado de la temperatura conociendo el valor de la cobertura. Por lo tanto, la valuación de opciones sobre temperatura permite optimar costos por consumo de energía (dada la correlación entre los precios de energía) para calentar o refrigerar. El equilibrio entre la demanda y la oferta de energía determina el costo de capital para calentar o enfriar y los precios de la energía dependen del clima (la demanda de energía para calefacción incrementa en el cuarto trimestre debido al frío y la demanda disminuye al final del invierno por el calor). El portafolio presenta dos dimensiones de política de ingeniería de costos de capital: primero, fijar el periodo de cobertura y segundo, tomar una posición larga en la opción europea sobre el índice de temperatura para cubrir los costos de capital por el consumo de energía que la empresa no está dispuesta a cubrir. El portafolio cuantifica el costo de capital por el consumo de energía que la empresa ahorra, minimizando el costo de capital por consumo de energía administrando el riesgo con la posición larga en la opción europea de compra sobre el índice de temperatura para minimizar la factura de los costos de energía para calentar o refrigerar. 
El costo de capital por el consumo de energía y la eficiencia de la política de costos dependen de la valuación de la opción europea de compra sobre la temperatura. El costo de capital por consumo de energía requiere de coberturas eficientes, que permiten a las empresas monitorear el consumo mientras preservan la competitividad. La dependencia energética derivada de la temperatura representa un riesgo operativo y las coberturas eficientes minimizan el costo de capital por el consumo de energía, permitiendo el crecimiento y el desarrollo económico. Las coberturas, por lo tanto, impulsan las preferencias hacia las valuaciones, que exhiben pertinencia y precios significativos.

El clima impacta la situación financiera y económica de empresas en diferentes sectores económicos (primario: agrícola, forestal, ganadero, minero pesquero, secundario: construcción, manufacturero, y terciario: comercio, servicios y transporte), la ingeniería financiera a través de los derivados climáticos es una herramienta utilizada en la administración de riesgos para la cobertura de pérdidas potenciales generadas por el clima; el Chicago Mercantil Exchange (CME) es pionero en la negociación de derivados climáticos donde la temperatura es una variable explicativa del consumo de energía y productos o servicios derivados, el Chicago Board of Trade (CBOT) negoció las primeras opciones sobre mercancías (commodities) en 1984 y ha introducido opciones catastróficas, por lo tanto, es importante que en México exista la posibilidad de coberturas de riesgos en el contexto de costos derivados por cambios climáticos porque son potencialmente significativas para los sectores económicos a raíz de la reforma energética; para la comercialización de derivados climáticos es necesario identificar riesgos por cubrir, modelos de valuación y aceptar la imposibilidad de transferir completamente los riesgos, por lo tanto, los mercados incompletos son adecuados porque aceptan propuestas para índices y modelos, innovando a través de la ingeniería financiera en coberturas y administración de riesgos climáticos.

Considine (2000) indica que la primera transacción en el mercado de derivados climáticos fue en Estados Unidos en 1997. Jain y Baile (2000) indican que se han realizado aproximadamente 3,000 contratos por 5 mil millones y medio de dólares en el mercado de derivados climáticos de Estados Unidos, y en Europa se han completado alrededor de 100 contratos climáticos por 30 millones de libras esterlinas. Brody et. al. (2002) indican que la temperatura se modela con el movimiento browniano y caracterizan la dinámica de la temperatura con un proceso Uhlenbeck y Ornstein (1930) para fijar el precio de dos tipos de reclamos contingentes: uno basado en grados de calentamiento y enfriamiento, y otro basado en la temperatura acumulada; y proponen expresiones analíticas para los pagos descontados esperados de estos derivados.

Alaton et. al. (2002) indican que el clima impacta en las actividades empresariales, el sector energético ha impulsado los derivados climáticos y el progreso de la administración de riesgos climáticos; las variables subyacentes son: temperatura, humedad, lluvia o nevadas 
y el subyacente común es la temperatura; los productores de energía han registrado que los precios están correlacionados con el clima y los derivados climáticos son una forma para cubrir riesgos; los mercados de capitales y el sector asegurador se han unido y el número de bonos catastróficos emitidos ha incrementado; el CBOT ha introducido opciones catastróficas y los derivados climáticos son la extensión lógica; los derivados climáticos permiten cubrir costos derivados de cambios climáticos. Los autores modelan precios para derivados climáticos con pagos contingentes en función de la temperatura usando datos históricos para sugerir un proceso estocástico browniano que describe la temperatura, obteniendo precios en un mercado incompleto. Presentan resultados numéricos utilizando una fórmula de aproximación y simulaciones Monte Carlo. Concluyen que el modelo implementado es una simplificación de la realidad y factible de mejora, e indican que aun cuando el modelo parece ajustar bien los datos de temperatura y que el modelo se apega a la realidad, aplicando un modelo para el proceso de volatilidad dinámica para pronósticos significativos en la valuación de derivados climáticos y con negociaciones en tiempo real se podría encontrar una estructura adecuada para los precios de mercado.

Benth (2003) propone una dinámica de precios libre de arbitraje para reclamos contingentes sobre temperatura con un proceso Uhlenbeck y Ornstein (1930) y propone expresiones explícitas para reclamos contingentes de tipo europeo y promedio. Platen y West (2004) proponen un enfoque para los precios de derivados climáticos comercializados en un mercado incompleto. Muestran que la metodología actuarial es un caso particular del concepto de precios justos. Plantean un modelo de tiempo discreto para aproximar las características históricas del clima. Benth et. al. (2011) indican que los derivados sobre el clima son diferentes a los derivados financieros porque el clima no puede ser negociado y, por lo tanto, no es replicado por otros instrumentos financieros. Analizan la estructura del precio de mercado en los mercados emergentes de Asia y descubrieron que las temperaturas asiáticas (Tokio, Osaka, Beijing, Teipei) son gaussianas en el sentido de que los procesos estocásticos están cerca de un proceso de Wiener. Manfredo y Richards (2009) indican que los derivados sobre el clima son una innovación financiera para la administración de riesgos, y cuando son utilizados derivados climáticos para cubrir riesgos, los administradores de riesgos enfrentan riesgos que surgen de la elección de la estación meteorológica donde se suscribe un contrato de derivados y el índice meteorológico subyacente. Muestran que la relación no lineal entre los rendimientos de las cosechas y el clima crea una cobertura específica para las opciones sobre el clima.

Musshoff et. al. (2009) indican que el clima es una fuente de incertidumbre en los cultivos, las fluctuaciones climáticas aumentan en el futuro por el cambio climático y los agricultores intentaron protegerse contra las variaciones de los rendimientos relacionadas con el clima mediante seguros, y que recientemente ha habido una discusión sobre el uso de derivados climáticos para cubrir riesgos volumétricos. Aun cuando los derivados climáticos muestran 
ventajas sobre los seguros, existe un mercado pequeño para estos productos en la agricultura. Analizan el rendimiento real y los datos climáticos del noreste de Alemania para cuantificar el efecto de reducción de riesgos que se logra en la producción de trigo con opciones de precipitación utilizando la simulación estocástica.

Nave Pineda y González Sánchez (2010) indican que las actividades económicas están expuestas a condiciones climáticas y que son relevantes para los sectores energético, agrícola, turístico y asegurador. Conjeturan que la demanda de productos de cobertura sobre el clima es creciente, impulsando los derivados climáticos y el desarrollo de la administración de riesgos climáticos. Sugieren que los mercados de derivados climáticos exhortan a estandarizar las condiciones de los contratos. Mencionan que los derivados sobre temperatura son los más utilizados porque la temperatura es una variable explicativa del consumo de energía. Analizan productos derivados sobre la temperatura aplicados al Aeropuerto Eldorado, ajustando la temperatura a un modelo Uhlenbeck y Ornstein (1930) de reversión a la media con un proceso de Wiener, comprueban su validez con simulación y realizan la valuación de varios productos derivados. Concluyen que los derivados climáticos son empleados para cubrir los estados de resultados de actividades económicas sensibles a factores climáticos y su presencia en mercados financieros confiere flexibilidad en la administración de riesgos de la actividad empresarial en general y a los sectores asegurador y eléctrico; el desarrollo y uso de productos derivados financieros exige el análisis del subyacente para una medición y valuación adecuada del riesgo por administrar; el modelo de Uhlenbeck y Ornstein (1930) presenta una adecuada bondad de ajuste sobre la muestra de datos diarios de 28 años (1979-2006) confirmando las propiedades de reversión a la media y volatilidad estacional por periodos mensuales empleando el modelo para la valuación de productos derivados sobre la temperatura usando técnicas de simulación y fórmulas cerradas que suponen un comportamiento gaussiano de las temperaturas mensuales.

Alva Vázquez y Sierra Juárez (2010) señalan que por la situación geográfica de México ocurren una variedad de fenómenos naturales cada año, generando cambios que causan desastres (terremotos, huracanes, inundaciones y sequías) con efectos en los recursos naturales y financieros. "El Niño" afecta la pesca en términos monetarios, sus efectos significaron una pérdida aproximada de $\$ 700,000,000$ en 1998 . El clima impacta en las actividades financieras, la lista de empresas expuestas al riesgo climático incluye: productores y consumidores de energía, cadenas de supermercados, industrias de diversión y recreación, e industrias agrícolas y pesqueras. Indican que en México es inexiste el mercado de derivados climáticos, y proponen un modelo para valuación de opciones climáticas para el sector pesquero del océano Pacífico mexicano, donde la variable fundamental es la temperatura del mar, con el objetivo de plantear un sistema de cobertura con datos históricos de la temperatura del mar en diferentes regiones para proponer un proceso estocástico que describe la evolución de la temperatura del océano. 
Concluyen que las primas de las opciones están dentro del intervalo del 10 al 20\% del valor nocional del contrato, por lo tanto, la existencia de opciones climáticas es de utilidad para la cobertura ante los cambios de clima en los océanos y que inciden en el medio ambiente, adaptando los pronósticos de índices fundamentales (temperatura, lluvia y nieve) usados en los derivados climáticos.

Goncu (2011) señala que los derivados climáticos son una forma de seguridad financiera con pagos contingentes condicionados a índices meteorológicos que ofrecen coberturas para el riesgo de cambios climáticos en términos de ingresos y costos, y que existe un potencial significativo para su uso porque aproximadamente un séptimo de la industria es sensible al clima. Explica que la agricultura está afectada significativamente por las variables climáticas. Analiza los derivados climáticos basados en los índices de temperatura en días de calor (HDD, acrónimo de Heating Degree Days) y en los índices de temperatura en días de frío (CDD, acrónimo de Cooling Degree Days) que son negociados en el CME. Indica que es el primer estudio para el precio de los derivados climáticos basados en la temperatura promedio diaria de las ciudades Beijing, Shanghai y Shenzhen utilizando un modelo dinámico con una función de volatilidad constante por periodos, donde las estimaciones de precios de las opciones son obtenidas con simulación Monte Carlo y métodos de aproximación analítica. Concluye que las opciones sobre HDD y CDD presentan un error relativo menor para el estimador Monte Carlo con respecto a la aproximación analítica conforme las opciones se encuentran más dentro de dinero, y que la fórmula de aproximación es menos confiable para los contratos sobre HDD en Shenzhen porque el supuesto de distribución es incumplido por las observaciones empíricas en algunas ocasiones, generando errores relativos mayores, y cuando la suposición de distribución subyacente es incumplida, la simulación de Monte Carlo y la aproximación producen resultados cercanos.

Chang y Tang (2016) indican que las opciones climáticas permiten a las compañías asegurarse contra fluctuaciones climáticas. Suponiendo autofinanciamiento y un conjunto correlacionado que sigue un movimiento browniano geométrico con un proceso de difusión con saltos presentan un modelo de valuación de opciones climáticas sobre temperatura con un movimiento browniano de reversión media, derivando una ecuación diferencial parcial bidimensional aplicando un método numérico fundamentado en una técnica de volumen finito combinada con la derivada lagrangiana y proporcionan ejemplos numéricos para una serie de opciones europeas de venta sobre los índices de grados de calentamiento. Tang y Chang (2016) presentan un modelo de valuación de opciones climáticas sobre la temperatura que sigue un movimiento de reversión de la media con un movimiento browniano de difusión con saltos suponiendo autofinanciación y derivando una ecuación integro diferencial parcial bidimensional para la valuación de una serie de opciones europeas de venta sobre el índice de grados de calentamiento aplicando técnicas numéricas. 
Groll et. al. (2016) analizan una metodología de valuación de derivados sobre temperatura con información prospectiva proporcionando un respaldo empírico para el marco teórico de los modelos consistentes para los pronósticos de la temperatura y aplican esta metodología realizando un análisis estadístico de los pronósticos meteorológicos y proponen un modelo de dos factores de Uhlenbeck y Ornstein (1930) con dos procesos gaussianos independientes que consideran la información prospectiva, valuando derivados sobre temperatura y calibrando el precio de riesgo de mercado. La potencia del modelo es comparada con modelos alternativos, confirmando que el precio de riesgo de mercado es por la especificación errónea de la información.

Huang et al. (2018) investigan el comportamiento de la temperatura para desarrollar un modelo ARFIMA Seasonal GARCH que modela los efectos estacionales, cíclicos, calentamiento global, y de memoria larga que proporciona una bondad de ajuste y precisión de pronóstico utilizando temperaturas promedio diarias en seis ciudades estadounidenses, analizan el comportamiento de la temperatura en los precios de los derivados sobre la temperatura, proponen la valuación numérica de opciones para los índices de calentamiento y enfriamiento a plazo y contratos de opciones con el modelo desarrollado que contribuye al desarrollo para los mercados de derivados sobre clima.

Dzupire et al. (2019) indican que los mercados de derivados climáticos son mercados incompletos porque los índices climáticos no son activos negociables, entonces, los métodos de valuación como Black y Scholes (1973) no son aplicables en la valuación de derivados climáticos, entonces, desarrollan un método para la valuación de una canasta de derivados meteorológicos sobre lluvia que es modelada como un proceso Gama estacionario transformado en un proceso browniano geométrico sobre la temperatura que es modelada con un proceso Uhlenbeck y Ornstein (1930) con un proceso de Wiener correlacionado con el de la lluvia en un mercado incompleto donde la preferencia de riesgo del agente económico es una función de utilidad exponencial y los precios son derivados con el principio de programación dinámica partiendo de los problemas de control óptimo estocástico y encuentran que la medida de indiferencia es igual a la medida física porque la correlación entre el mercado de capitales y el clima es inexistente. El precio justo del derivado es mayor que el precio de indiferencia del vendedor y menor que el precio de indiferencia del comprador por la viabilidad del mercado sin oportunidades de arbitraje. Concluyen que el modelo desarrollado puede aplicar una función de utilidad diferente, lo que significa una preferencia de riesgo diferente y dado que los derivados climáticos son negociados extrabursátilmente, entonces, las valuaciones son comparadas con enfoques actuariales.

La hipótesis propuesta en este trabajo es que es posible innovar en el modelado de la temperatura para la valuación de productos derivados para la cobertura de costos derivados por los riesgos de cambios en la temperatura, el objetivo es proponer un modelo de valuación de 
opciones sobre la temperatura y probar la idoneidad del modelo a través de distribuciones que modelan eventos extremos y asimétricos que generan impactos financieros y económicos de cuantías superiores que los esperados por el modelo gaussiano Uhlenbeck y Ornstein (1930), y que además satisfacen el teorema del límite central generalizado en dominios de atracción $\alpha$-estables que estiman más adecuadamente riesgos de proyectos con ingeniería financiera y administración de riesgos utilizando la teoría de valuación de productos derivados climáticos en mercados incompletos con el parámetro de estabilidad.

El trabajo está organizado de la forma siguiente: en la sección 2 se presenta el comportamiento estacional de las temperaturas, son estimados los estadísticos descriptivos, es presentado un análisis cualitativo de la distribución empírica, es estimado un modelo de regresión lineal simple con tendencia positiva y significativo, y un modelo determinista de reversión a la media con ordenada al origen, tendencia positiva y amplitud que son significativas y que mejora al modelo de regresión lineal simple, es propuesto un modelo estocástico de reversión a la media con un proceso estocástico $\alpha$-estable, es realizado una análisis mensual de las temperaturas, son estimados los parámetros $\alpha$-estables mensuales, son realizadas pruebas de bondad de ajuste y no se rechazan a las distribuciones $\alpha$-estables para las temperaturas promedio mensuales, se propone la estimación del parámetro de reversión a la media con procesos estocásticos $\alpha$-estables, y se propone la solución de la ecuación diferencial estocástica; en la sección 3 se propone el modelo para la valuación de opciones europeas de compra sobre los índices de grados de calentamiento y enfriamiento con distribuciones $\alpha$-estables, son estimados los índices de temperatura de 2010, y son valuadas las opciones de compra sobre los índices; en la sección 4 se presentan las conclusiones, y por último la las referencias bibliográficas.

\section{Modelado de la temperatura promedio}

La temperatura es un factor exógeno que incide en la valuación de los derivados climáticos para cubrir costos derivados por cambios en la temperatura. Los datos utilizados son las temperaturas mínimas, máximas y promedio diarias del observatorio central de Tacubaya (CNASMN-SCDI, climatología estadística, unidad: ${ }^{\circ} \mathrm{C}$, estación: 9048, estado: Ciudad de México, municipio: Miguel Hidalgo, organismo: CONAGUA-DGE, latitud: 9²4'13" Norte, longitud: 99 11 '46" Oeste, altitud: 2,308.6 msnm) durante el período comprendido del 01/01/1958 al $31 / 12 / 2018$ con un total de 22,280 observaciones. Los datos son utilizados para ajustar un modelo que describe el subyacente de los derivados climáticos, proponiendo que los sectores que utilizan coberturas tienen información oportuna y veraz que los observatorios garantizan para que la existencia de índices permita a inversionistas y emisores modelar, validar y cuantificar los riesgos climáticos con información oficial para la operación en mercados nacionales e internacionales. La información del observatorio de Tacubaya contiene temperaturas diarias: 
mínimas y máximas en grados centígrados, y a partir de ambas es calculada la temperatura promedio de la forma siguiente:

$$
\operatorname{pro}\left(T_{k}\right)=\frac{\operatorname{mín}\left(T_{k}\right)+\operatorname{máx}\left(T_{k}\right)}{2}
$$

donde $\operatorname{pro}\left(T_{k}\right)$ es la temperatura promedio, $\operatorname{mín}\left(T_{k}\right)$ y $\operatorname{máx}\left(T_{k}\right)$ son las temperaturas mínimas y máximas. El comportamiento de las temperaturas es presentado en la Figura 1.

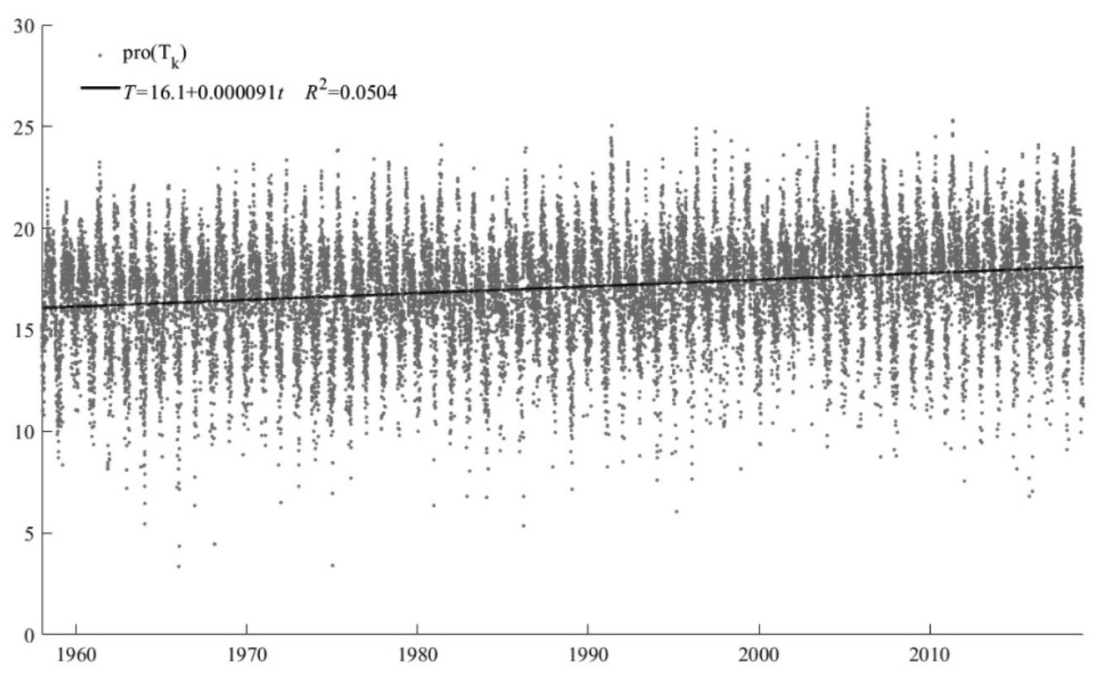

Figura 1. Comportamiento de las temperaturas promedio

Fuente: Elaboración propia con datos del observatorio central de Tacubaya

La Figura 1 indica que las temperaturas promedio oscilan entre $3.35^{\circ} \mathrm{C}$, que se presentó el 8 de enero de 1964 , y $25.9^{\circ} \mathrm{C}$, que se presentó el 10 de mayo de 2006 , los periodos más fríos se presentan entre octubre y marzo, los periodos más cálidos se presentan entre marzo y junio, con un promedio de $17.08^{\circ} \mathrm{C}$, una desviación estándar de $2.60^{\circ} \mathrm{C}$ y una tendencia positiva significativa. La estimación de los estadísticos descriptivos de las temperaturas promedio es presentada en la Tabla 1 . 
Tabla 1

Estadísticos descriptivos de las temperaturas promedio

\begin{tabular}{ccccccc}
\hline Temperatura & Mínimo & Máximo & Promedio & Desviación & Asimetría & Curtosis \\
\hline Promedio & 3.35 & 25.90 & 17.08 & 2.60 & -0.36 & 3.46 \\
\hline
\end{tabular}

Fuente: Elaboración propia con datos del observatorio central de Tacubaya

La Tabla 1 indica que las temperaturas promedio oscilan entre $3.35^{\circ} \mathrm{C}$ y $25.9^{\circ} \mathrm{C}$ con un promedio de $17.08^{\circ} \mathrm{C}$, una volatilidad de $2.60^{\circ} \mathrm{C}$, el coeficiente de asimetría negativa indica que las temperaturas promedio presentan una distribución asimétrica con una pendiente menor en el extremo izquierdo que en el extremo derecho de la moda $X=18$, y el coeficiente de curtosis mayor que tres indica que la distribución de las temperaturas promedio son leptocúrticas con respecto a la distribución gaussiana, por lo tanto, las temperaturas presentan distribuciones leptocúrticas y asimétricas. El histograma de frecuencias absolutas de las temperaturas promedio es presentado en la Figura 2.

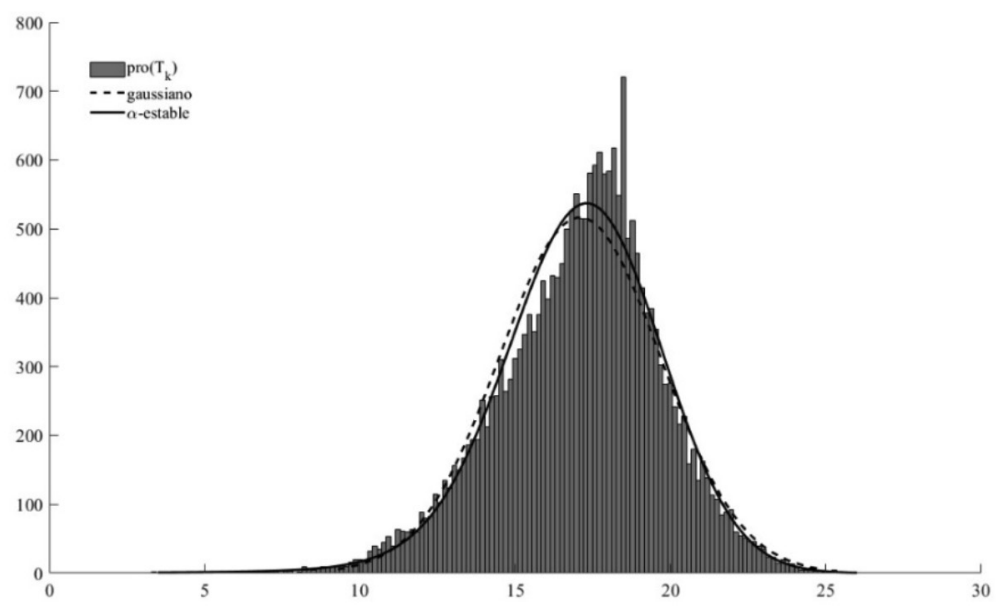

Figura 2. Frecuencias de las temperaturas promedio

Fuente: Elaboración propia con datos del observatorio central de Tacubaya

La Figura 2 indica que las temperaturas promedio presentan una distribución con asimetría negativa, es decir, las temperaturas promedio menores que la moda para datos agrupados, $X=18.10$, representan el $60.76 \%$ de las temperaturas promedio y presentan una distribución leptocúrtica con respecto a la distribución gaussiana, es decir, los extremos derecho e izquierdo de la distribución empírica presentan frecuencias mayores que las esperadas por la distribución gaussiana. 
Los valores extremos (mínimos o máximos) de las temperaturas promedio representan un impacto financiero y económico superior al costo de producción o servicio derivado de la leptocurtosis y de la asimetría porque la distribución gaussiana es inapropiada para modelar los valores extremos y la asimetría porque es simétrica y mesocúrtica. Los acontecimientos climáticos que impactan a las empresas y que la distribución gaussiana es incapaz de modelar adecuadamente indican la importancia de valuar coberturas apropiadas ante los cambios climáticos generados por factores exógenos entre los que se encuentran: actividades humanas como agricultura, ganadería, deforestación, y el uso de combustibles fósiles como: carbón, petróleo, gasolina, diésel, turbosina y gas, que generan cambios atmosféricos y biofísicos que producen gases de efecto invernadero como: metano $\left(\mathrm{NH}_{4}\right)$, óxido de carbono $\left(\mathrm{CO}_{2}\right)$, ozono $\left(\mathrm{O}_{3}\right)$, y óxidos de nitrógeno ( $\mathrm{NO}, \mathrm{NO}_{2}, \mathrm{~N}_{2} \mathrm{O}, \mathrm{N}_{2} \mathrm{O}_{3}, \mathrm{~N}_{2} \mathrm{O}_{4}, \mathrm{~N}_{2} \mathrm{O}_{5}$ ), además de factores como la radiación solar; factores que están fuera del análisis y objetivos de este trabajo que está centrado en la valuación de opciones sobre índices de temperatura, subyacente que presenta una tendencia positiva significativa en los años recientes (1958-2018). Las estimaciones de la ordenada al origen y la pendiente de las temperaturas promedio son presentadas en la Tabla 2.

Tabla 2

Estimación de la ordenada y pendiente de las temperaturas promedio

\begin{tabular}{ccccccc}
\hline Temperatura & $\beta_{0}$ & $\beta_{1}$ & $R^{2}$ & $P\left(t_{\beta_{0}}\right)$ & $P\left(t_{\beta_{1}}\right)$ & $P(F)$ \\
\hline Promedio & 16.1 & 0.000091 & 0.0504 & 0.0000 & $2.64 \mathrm{E}-252$ & $2.64 \mathrm{E}-252$ \\
\hline
\end{tabular}

Fuente: Elaboración propia con datos del observatorio central de Tacubaya

La Tabla 2 indica que la ordenada de las temperaturas promedio es $16.11^{\circ} \mathrm{C}$, la pendiente es 0.000091, y están representada por la ecuación y la línea continua de la Figura 1; con un pronóstico de $18.11^{\circ} \mathrm{C}$ para finales del año 2018; el coeficiente de determinación es 0.0504 . Las probabilidades de los estadísticos $t$ y $F$ son significativas para las temperaturas promedio, por lo tanto, los incrementos de las temperaturas son significativos y es esperado un incremento de $2.04^{\circ} \mathrm{C}$.

\section{Modelo determinista de reversión a la media}

La Figura 1 muestra una variación periódica de las temperaturas con periodos anuales, con tendencias positivas y significativas, con factores exógenos como causales, entonces, un modelo determinista de reversión a la media es estimado para modelar las temperaturas:

$$
T_{t}^{m}=A+B t+C \operatorname{sen}(\omega t+\varphi)
$$


donde $T_{t}^{m}$ es la temperatura, ${ }^{t}$ representa el tiempo, la función seno permite modelar la variación periódica anual, por lo tanto, $\omega=2 \pi(365)^{-1}, \varphi$ representa un ángulo fase para los periodos fríos y cálidos que ocurren en fechas diferentes al inicio o final del año, $A, B, C$ y $\varphi$ son los estimadores que ajustan las temperaturas a través del método de mínimos cuadrados, empleando la identidad:

$$
\|(a, b)\| \operatorname{sen}\left(\omega t+\arctan \left(b a^{-1}\right)\right)=a \operatorname{sen}(\omega t)+b \cos (\omega t)
$$

entonces, el modelo transformado s:

$$
T=\beta_{0}+\beta_{1} t+\beta_{2} \operatorname{sen}(\omega t)+\beta_{3} \cos (\omega t)
$$

y los parámetros para las temperaturas promedio son:

$$
A=\beta_{0}, \quad B=\beta_{1}, \quad C=\sqrt{\beta_{2}^{2}+\beta_{3}^{2}}, \quad \varphi=\arctan \left(\beta_{3} \beta_{2}^{-1}\right)
$$

donde $A$ es la ordenada, $B$ es la pendiente, $C$ es la amplitud de la función de variación periódica anual y $\varphi$ es el ángulo fase de los periodos fríos y cálidos. La estimación de parámetros del modelo determinista de reversión a la media, por medio de la ecuación , es presentada en la Tabla 3.

\begin{tabular}{|c|c|c|c|c|c|c|c|c|c|c|}
\hline Temperatura & $\beta_{0}$ & $\beta_{1}$ & $\beta_{2}$ & $\beta_{3}$ & $R^{2}$ & $P\left(t_{\beta_{0}}\right)$ & $P\left(t_{\beta_{1}}\right)$ & $P\left(t_{\beta_{2}}\right.$ & $P\left(t_{\beta_{3}}\right)$ & $P(F)$ \\
\hline Promedio & 16.1 & $0.092 \mathrm{E}-5$ & 0.8 & -2.2 & 0.46 & 0.00 & 0.00 & 0.00 & 0.00 & 0.00 \\
\hline
\end{tabular}

\section{Tabla 3}

Estimación de parámetros del modelo transformado

Fuente: Elaboración propia con datos del observatorio central de Tacubaya

La Tabla 3 indica que la ordenada del modelo determinista de reversión a la media es $16.1^{\circ} \mathrm{C}$ y se aproxima a la ordenadas del modelo de regresión lineal simple; la pendiente es 0.000092; las pendientes, $\beta_{2}$ y $\beta_{3}$, para calcular la amplitud de la variación periódica, son significativas; el coeficiente de determinación es 0.46 , entonces, el modelo determinista de reversión a la media mejora al modelo lineal simple, las probabilidades de los estadísticos $F$ indican que el modelo determinista de reversión a la media es significativo. La estimación de parámetros del modelo determinista de reversión a la media, con las ecuaciones y, son presentadas en la Tabla 4. 
Tabla 4

Estimación de parámetros del modelo determinista de reversión a la media

\begin{tabular}{ccccr}
\hline Temperatura & $A$ & $B$ & $C$ & $\varphi$ \\
\hline Promedio & 16.1 & 0.000092 & 2.35 & -1.22 \\
\hline
\end{tabular}

Fuente: Elaboración propia con datos del observatorio central de Tacubaya

La Tabla 4 indica que la ordenada del modelo determinista de reversión a la media es $16.1^{\circ} \mathrm{C}$; la pendiente es 0.000092 ; la amplitud de la función de variación periódica es 2.35 , es decir, la diferencia entre temperaturas promedio es $4.7^{\circ} \mathrm{C}$. Los resultados de las pruebas de diagnóstico para los residuales de los modelos deterministas son presentados en la Tabla 5.

\section{Tabla 5}

Pruebas de diagnóstico de los modelos deterministas

\begin{tabular}{ccccccc}
\hline & \multicolumn{5}{c}{$T=\beta_{0}+\beta_{1} t$} & \\
Prueba & Estadístico & Valor & $P(T>t)$ & Estadístico & Valor & $P(T>t)$ \\
\hline Correlación & $\chi_{L M}^{2}(1)$ & $16,927.6$ & 0.0000 & $\chi_{L M}^{2}(1)$ & $1,3375.3$ & 0.0000 \\
Correlación & $\chi_{L M}^{2}(2)$ & $16,941.4$ & 0.0000 & $\chi_{L M}^{2}(2)$ & $13,375.3$ & 0.0000 \\
Heterocedasticidad & $\chi_{B P G}^{2}(1)$ & 0.0 & 0.9787 & $\chi_{B P G}^{2}(3)$ & 419.1 & 0.0000 \\
Heterocedasticidad & $\chi_{W}^{2}(2)$ & 20.5 & 0.0000 & $\chi_{W}^{2}(9)$ & 558.1 & 0.0000 \\
Especificación & $F(1, n-k-1)$ & 3.4 & 0.0649 & $F(1, n-k-1)$ & 493.0 & 0.0000 \\
Normalidad & $\chi_{J B}^{2}(2)$ & 760.1 & 0.0000 & $\chi_{L M}^{2}(2)$ & $1,190.5$ & 0.0000 \\
\hline
\end{tabular}

Fuente: Elaboración propia con datos del observatorio central de Tacubaya

La Tabla 5 presenta los resultados de las pruebas de incorrecta especificación aplicadas a los modelos estimados. Las pruebas aplicadas son Pruebas Breusch y Godfrey para correlación serial con $\chi_{L M}^{2}(1)_{\mathrm{V}} \quad \chi_{L M}^{2}(2)$, Breusch, Pagan y Godfrey y White para heterocedasticidad con $\chi_{B P G}^{2}(k-1)$ y $\chi_{W}^{2}(l)$, Ramsey para especificación con $F(1, n-k-1)$, Jarque y Bera para normalidad con $\chi_{J B}^{2}(2)$. Los resultados indican que el modelo lineal simple supera las pruebas de Breusch y Godfrey para correlación serial y para heterocedasticidad al 5\%. El modelo 
determinista de reversión a la media no supera ninguna de las pruebas aplicadas. Los resultados son atribuidos a la volatilidad inherente a las temperaturas diarias, que es muy volátil en algunos periodos, a la presencia de cambios estructurales de la temperatura o a cambios de régimen como el cambio climático. El modelo determinista de reversión a la media del 1 de enero de 2009 al 31 de diciembre de 2018 se presenta en la Figura 3.

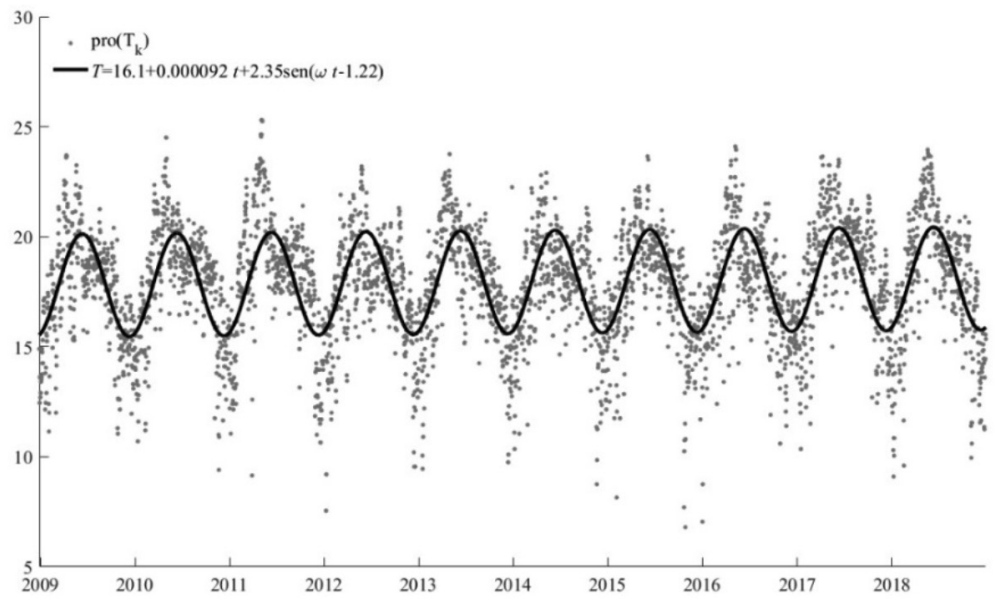

Figura 3. Modelo determinista de reversión a la media de las temperaturas Fuente: Elaboración propia con datos del observatorio central de Tacubaya

La Figura 3 presenta el ajuste del modelo determinista de reversión a la media con las temperaturas promedio observadas durante los últimos diez años y se observa que captura más adecuadamente el periodo de oscilación anual y la tendencia positiva que el modelo lineal simple. Por lo tanto, se tiene un modelo determinista de reversión a la media para modelar las temperaturas.

\section{Modelo estocástico de reversión a la media}

El comportamiento de las temperaturas presenta una variación periódica con tendencia positiva y una variación estocástica alrededor de la variación periódica, entonces, la primera innovación presentada es la ecuación diferencial estocástica:

$$
\mathrm{d} T_{t}=a\left(T_{t}^{m}-T_{t}\right) \mathrm{d} t+\gamma_{t} \mathrm{~d} Z_{t}
$$


donde $a$ es la tasa de reversión a la media, $\gamma_{t}$ es el parámetro de escala y $Z_{t}$ es un proceso_a-estable estándar y autosimilar. La solución de la ecuación, en el caso particular donde ${ }_{t}$ es un proceso de Wiener, es un proceso Uhlenbeck y Ornstein (1930). En el largo plazo, la ecuación no presenta reversión a la media, entonces, incluvendo la derivada:

$$
\frac{\mathrm{d} T_{t}^{m}}{\mathrm{~d} t}=B+\omega C \cos (\omega t+\varphi)
$$

a la tendencia de la ecuación, es obtenido un proceso de reversión a la media porque las temperaturas modeladas por $T_{t}^{m}$ presentan variaciones periódicas que ajustan la tendencia para que la ecuación diferencial estocástica presente reversión a la media en el largo plazo. Con la condición inicial $T_{s}=x$ se obtiene la ecuación diferencial estocástica para modelar la temperatura:

$$
\mathrm{d} T_{t}=\left(\frac{\mathrm{d} T_{t}^{m}}{\mathrm{~d} t}+a\left(T_{t}^{m}-T_{t}\right)\right) \mathrm{d} t+\gamma_{t} \mathrm{~d} Z_{t} \quad \forall t>s
$$

con la solución:

$$
T_{t}=\left(T_{s}-T_{s}^{m}\right) \exp (-a(t-s))+T_{t}^{m}+\int_{s}^{t} \exp (-a(t-\tau)) \gamma_{\tau} \mathrm{d} Z_{\tau}
$$

$$
\text { donde } T_{t}^{m}=A+B t+C \operatorname{sen}(\omega t+\varphi) \text {. }
$$

\section{Análisis mensual de las temperaturas promedio}

Con fundamento en los resultados previos es realizado un análisis mensual de las temperaturas promedio. Las estimaciones de los parámetros $\alpha$-estables de los periodos mensuales son presentadas en la Tabla 6.

La Tabla 6 presenta los parámetros $\alpha$-estables. Los parámetros de estabilidad indican que las distribuciones de las temperaturas promedio mensuales son leptocúrticas, excepto mayo, junio y agosto para es estadístico $A^{2}$ y de abril hasta agosto para el estadístico $W^{2}$ . Los parámetros de asimetría indican que las distribuciones se extienden hacia el extremo izquierdo más lento que hacia el extremo derecho, excepto abril, mayo y junio. Los parámetros de escala indican que las distribuciones presentan dispersión estacional. Los parámetros de localización indican que las distribuciones presentan cambios estacionales. Concluyendo que las temperaturas mensuales son leptocúrticas y asimétricas negativas en la mayoría de 
los meses, entonces, es necesario probar la pertinencia de las distribuciones $\alpha$-estables para modelar las temperaturas mensuales. El análisis cuantitativo para probar la hipótesis de que las temperaturas mensuales presentan distribuciones gaussianas con las pruebas de bondad de ajuste Anderson y Darling (AD), y Cramér von Mises (CvM) es presentado en la Tabla 7.

Tabla 6

Estimación de parámetros $\alpha$-estables de las temperaturas mensuales

\begin{tabular}{ccccc}
\hline Periodo & $a$ & $\beta$ & $\gamma$ & $\delta$ \\
\hline Enero & 1.78 & -1.00 & 1.26 & 14.1 \\
Febrero & 1.84 & -1.00 & 1.41 & 15.5 \\
Marzo & 1.84 & -1.00 & 1.38 & 17.8 \\
Abril & 2.00 & 0.28 & 1.52 & 19.2 \\
Mayo & 2.00 & 0.54 & 1.47 & 19.6 \\
Junio & 2.00 & 0.10 & 1.24 & 18.9 \\
Julio & 1.97 & -1.00 & 0.93 & 17.8 \\
Agosto & 1.97 & -1.00 & 0.81 & 18.0 \\
Septiembre & 1.82 & -1.00 & 1.03 & 17.4 \\
Octubre & 1.82 & -0.99 & 1.32 & 16.5 \\
Noviembre & 1.78 & -1.00 & 1.26 & 15.3 \\
Diciembre & 1.85 & -1.00 & 1.25 & 14.2 \\
\hline
\end{tabular}

Fuente: Elaboración propia con datos del observatorio central de Tacubaya

La Tabla 7 presenta el estadístico $A^{2}$ y el nivel de significación de la prueba $\mathrm{AD}$, entonces, es rechazada la hipótesis de que las temperaturas presentan distribuciones gaussianas en 9 de los 12 periodos mensuales estudiados, es decir, $75 \%$, excepto las temperaturas promedio de mayo, junio y agosto, por lo tanto, es injustificable la hipótesis gaussiana para modelar las temperaturas. El estadístico $W^{2}$ y el nivel de significación de la prueba CvM rechaza la hipótesis de que las temperaturas presentan distribuciones gaussianas en 7 de los 12 periodos mensuales estudiados, es decir, 58.33\%, excepto las temperaturas promedio de abril hasta agosto, por lo tanto, también es injustificable la hipótesis gaussiana para modelar las temperaturas. El análisis cuantitativo para probar la hipótesis de que las temperaturas mensuales presentan distribuciones $\alpha$-estables con las pruebas de bondad de ajuste AD, y CvM son presentadas en la Tabla 8. 
Tabla 7

Pruebas AD y CvM para distribuciones gaussianas

\begin{tabular}{ccccc}
\hline Periodo & $A^{2}$ & $P\left(A^{2}>a^{2}\right)$ & $W^{2}$ & $P\left(W^{2}>w^{2}\right)$ \\
\hline Enero & 11.37 & 0.0005 & 1.82 & 0.0000 \\
Febrero & 6.92 & 0.0005 & 1.15 & 0.0010 \\
Marzo & 7.51 & 0.0005 & 1.15 & 0.0000 \\
Abril & 2.75 & 0.0005 & 0.38 & 0.0760 \\
Mayo & 0.44 & 0.2911 & 0.07 & 0.7260 \\
Junio & 0.59 & 0.1278 & 0.09 & 0.6530 \\
Julio & 1.12 & 0.0062 & 0.21 & 0.2800 \\
Agosto & 0.73 & 0.0574 & 0.12 & 0.4730 \\
Septiembre & 8.75 & 0.0005 & 1.38 & 0.0000 \\
Octubre & 9.77 & 0.0005 & 1.63 & 0.0000 \\
Noviembre & 10.27 & 0.0005 & 1.69 & 0.0000 \\
Diciembre & 8.19 & 0.0005 & 1.43 & 0.0000 \\
\hline
\end{tabular}

Fuente: Elaboración propia con datos del observatorio central de Tacubaya

Tabla 8

Pruebas AD y CvM para distribuciones $\alpha$-estables

\begin{tabular}{ccccc}
\hline Periodo & $A^{2}$ & $P\left(A^{2}>a^{2}\right)$ & $W^{2}$ & $P\left(W^{2}>w^{2}\right)$ \\
\hline Enero & 0.46 & 0.7831 & 0.05 & 0.8550 \\
Febrero & 1.58 & 0.1585 & 0.25 & 0.1780 \\
Marzo & 0.27 & 0.9584 & 0.03 & 0.9600 \\
Abril & 2.75 & 0.0368 & 0.38 & 0.0670 \\
Mayo & 0.44 & 0.8046 & 0.07 & 0.7240 \\
Junio & 0.59 & 0.6609 & 0.09 & 0.6700 \\
Julio & 0.62 & 0.6322 & 0.11 & 0.5050 \\
Agosto & 0.49 & 0.7551 & 0.08 & 0.6700 \\
Septiembre & 0.49 & 0.7598 & 0.06 & 0.7950 \\
Octubre & 1.52 & 0.1725 & 0.26 & 0.1910 \\
Noviembre & 1.11 & 0.3035 & 0.18 & 0.3040 \\
Diciembre & 1.69 & 0.1375 & 0.29 & 0.1350 \\
\hline
\end{tabular}

Fuente: Elaboración propia con datos del observatorio central de Tacubaya 
La Tabla 8 presenta los estadísticos $A^{2}$ y $W^{2}$, los niveles de significación de las pruebas $\mathrm{AD}$ y $\mathrm{CvM}$, entonces, la hipótesis de que las temperaturas presentan distribuciones $\alpha$-estables no es rechazada en la totalidad de los 12 periodos mensuales estudiados, es decir, 100\%, por lo tanto, la aplicación de las distribuciones $\alpha$-estables está justificada para modelar las temperaturas mensuales. De conformidad con los resultados presentados en las Tablas 6, 7 y 8 , se concluye que es factible modelar las temperaturas y proponer un modelo para valuar opciones mensuales sobre índices de temperatura en mercados incompletos con distribuciones $\alpha$-estables para generar coberturas anuales.

\section{Estimación del parámetro de reversión a la media}

Las observaciones de las temperaturas son diarias, entonces, para estimar el parámetro de reversión a la media se propone un estimador eficiente suponiendo que el parámetro de escala es constante en periodos mensuales, por lo tanto:

$$
G(\hat{a})=\sum_{k=1}^{n} \frac{T_{k}^{m}-T_{k}}{\gamma_{k_{m}}^{\alpha}}\left(T_{k+1}-T_{k+1}^{m}-\left(T_{k}-T_{k}^{m}\right) \exp (-a)\right)=0
$$

donde $\gamma_{k_{m}}$ es el parámetro de dispersión mensual de las temperaturas para $m=1,2, \mathrm{~K}, 12$. Por lo tanto, la segunda innovación es la estimación del parámetro de reversión a la media:

$$
\hat{a}=\ln \left(\sum_{k=1}^{n} \frac{T_{k}^{m}-T_{k}}{\gamma_{k_{m}}^{\alpha}}\left(T_{k}-T_{k}^{m}\right)\right)-\ln \left(\sum_{k=1}^{n-1} \frac{T_{k}^{m}-T_{k}}{\gamma_{k_{m}}^{\alpha}}\left(T_{k+1}-T_{k+1}^{m}\right)\right)
$$

donde $T_{t}^{m}=A+B t+C \operatorname{sen}(\omega t+\varphi)$. La estimación del parámetro de reversión a la medía, por medio de la ecuación , es presentada en la Tabla 9.

Tabla 9

Estimación del parámetro de reversión a la media

\begin{tabular}{cc}
\hline Temperatura & $a$ \\
\hline Promedio & 0.264817 \\
\hline
\end{tabular}

Fuente: Elaboración propia con datos del observatorio central de Tacubaya 
La Tabla 9 presenta el estimador de reversión a la media que indica la velocidad con las que las temperaturas promedio regresan a la media, el resultado es congruente con los resultados presentados por Alaton et al. (2002) y Alva Vázquez y Sierra Juárez (2010). La tercera innovación, de acuerdo con el Ejemplo 3.6.4 de Samorodnitsky y Taqqu (1994), que es presentada es la solución de la ecuación es:

$$
T_{t}=\left(T_{s}-T_{s}^{m}\right) \exp (-a(t-s))+T_{t}^{m}+\gamma_{t_{m}}\left(\frac{1-\exp \left(-\alpha_{t_{m}} a(t-s)\right)}{\alpha_{t_{m}} a}\right)^{\frac{1}{\alpha_{t_{m}}}} Z_{t-s}(10)
$$

donde $Z_{t-s}$ son variables aleatorias $\alpha$-estables estandarizadas e independientes, entonces, el modelo estocástico de reversión a la media propuesto es:

$$
T_{t}=\left(T_{t-1}-T_{t-1}^{m}\right) \exp (-a \Delta t)+T_{t}^{m}+\gamma_{t_{m}}\left(\frac{1-\exp \left(-\alpha_{t_{m}} a \Delta t\right)}{\alpha_{t_{m}} a}\right)^{\frac{1}{\alpha_{t_{m}}}} Z_{t}
$$

donde $Z_{t} \sim S_{1}(\alpha, \beta)$

El modelo estocástico de reversión a la media de las temperaturas del año 2019 utilizando el promedio de 30,000 simulaciones para cada día es presentado en la Figura 4.

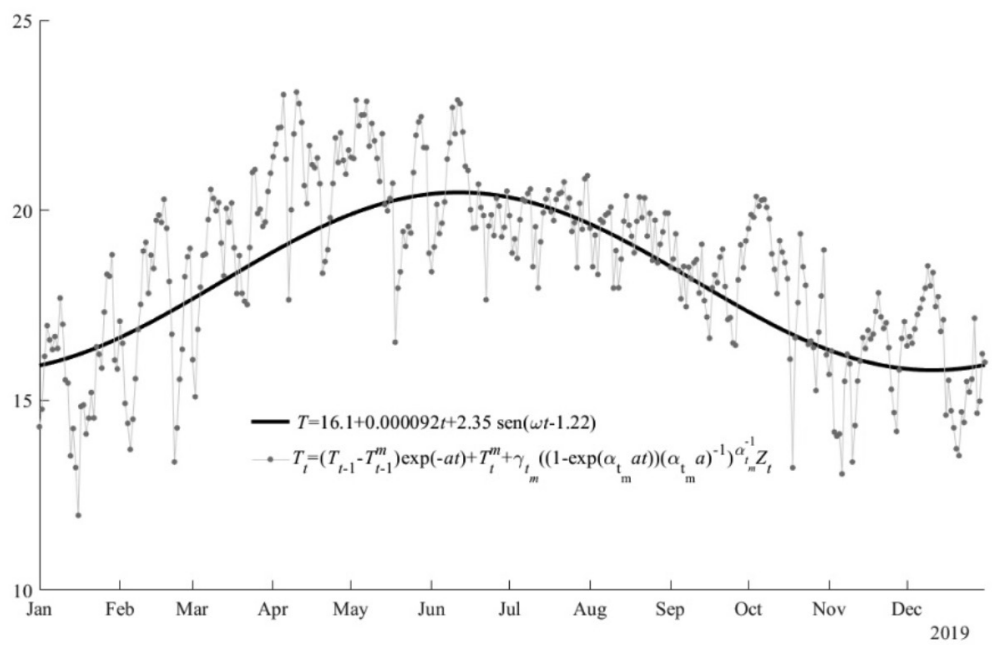

Figura 4. Modelo estocástico de reversión a la media de las temperaturas 2011

Fuente: Elaboración propia con datos del observatorio central de Tacubaya 
La Figura 4 presenta el comportamiento del modelo estocástico de reversión a la media utilizando el promedio diario de 30,000 simulaciones de variables aleatorias $\alpha$-estables estandarizadas e independientes para el año 2019 y se observa que el proceso aleatorio alrededor del proceso determinista de reversión a la media replica las temperaturas y, en su caso, permite la simulación de temperaturas del año siguiente.

\section{Derivados climáticos}

El mercado de derivados climáticos permite a las empresas obtener coberturas por riesgos derivados de cambios en la temperatura. La industria energética impulsó el crecimiento de los derivados climáticos, consumando la negociación de derivados climáticos en el CME en 1997, por lo tanto, es importante que en México existan coberturas de riesgos en el contexto de costos derivados por cambios climáticos. La reforma energética genera oportunidades de comercializar, en México, derivados climáticos considerando niveles de comercialización, riesgos por cubrir, modelos de valuación y aceptar la imposibilidad de transferir completamente los riesgos, por lo tanto, los mercados incompletos son la propuesta adecuada de modelos para índices, innovando con la ingeniería financiera: la cobertura y la administración de riesgos climáticos.

\section{Valuación de opciones de compra sobre la temperatura}

Suponiendo que el precio de riesgo de mercado es constante y que la empresa compra un activo libre de riesgo que es invertido a la tasa de interés constante $i$, y compran un contrato que por cada grado Celsius paga una unidad monetaria, entonces, bajo una medida equivalente, el proceso de precios satisface la ecuación . Los derivados climáticos sobre temperatura están basados en los grados de calentamiento o enfriamiento diarios. El pago de liquidación de una opción europea de compra sobre el índice de calentamiento diario es:

$$
H_{\tau}=N \operatorname{máx}\left(H_{T}-S, 0\right)=N \operatorname{máx}\left(\sum_{k=1}^{n} \operatorname{máx}\left(T_{0}-T_{k}, 0\right)-S, 0\right)
$$

donde $H_{\mathrm{t}}$ es el pago contingente y es parecido a una opción asiática de promedio aritmético, $N$ es el costo por grado de calentamiento diario, $H_{T}$ es el índice de los grados de calentamiento diario, $T_{0}$ es la temperatura de referencia, usualmente $18^{\circ} \mathrm{C}$, entonces, si la temperatura promedio es inferior a $18^{\circ} \mathrm{C}$, es necesario utilizar energía para aumentar la temperatura, por lo tanto, la temperatura de referencia es modificada de acuerdo a las necesidades de cobertura. El pago de liquidación de una opción europea de compra sobre el índice de enfriamiento diario es: 


$$
C_{\tau}=N \operatorname{máx}\left(C_{T}-S, 0\right)=N \operatorname{máx}\left(\sum_{k=1}^{n} \operatorname{máx}\left(T_{k}-T_{0}, 0\right)-S, 0\right)
$$

donde $C_{\mathrm{t}}$ es el pago contingente, $N$ es el costo por grado de enfriamientos diario, $C_{T}$ es el índice de enfriamiento diario, $T_{0}$ es la temperatura de referencia, $18^{\circ} \mathrm{C}$, entonces, si la temperatura promedio es superior a $18^{\circ} \mathrm{C}$, es necesario utilizar energía para disminuir la temperatura, por lo tanto, la temperatura de referencia es modificada de acuerdo con las necesidades de cobertura. La cuarta innovación presentada es que si el proceso $H_{\tau} \sim S_{1}(\alpha, \beta, \gamma, \delta)$, entonces, $H_{\tau}=H_{0}+\gamma Z_{\tau}$ y, entonces, el precio de la opción europea de compra sobre $H_{T}$ es:

$$
c\left(\tau, H_{\tau}\right)=\exp (-i \tau) \mathrm{E}\left(\operatorname{máx}\left(H_{\tau}-S, 0\right) \mid F_{\tau}\right)=\exp (-i \tau) \int_{S}^{\infty}\left(H_{\tau}-S\right) f_{Y_{\tau}}\left(y_{\tau}, \alpha, \beta, \gamma\right) \mathrm{d} Y_{\tau}
$$

donde $\tau=T-t$ es el tiempo remanente y el modelo gaussiano $H_{\tau} \sim S_{1}(2,0, \sigma, \mu)$ es un caso particular del modelo $\alpha$-estable. Estandarizando la función de distribución:

$$
c\left(\tau, H_{\tau}\right)=\exp (-i \tau) \int_{\frac{S-H_{0}}{\gamma}}^{\infty}\left(H_{\tau}-S\right) f_{Y_{\tau}}\left(y_{\tau}, \alpha, \beta\right) \mathrm{d} Y_{\tau}=\exp (-i \tau) \int_{-d}^{\infty}\left(H_{\tau}-S\right) f_{Y_{\tau}}\left(y_{\tau}, \alpha, \beta\right) \mathrm{d} Y_{\tau}
$$

Sustituyendo $H_{\tau}=H_{0}+\gamma Z_{\tau}$ y por el Teorema 2.4.1 de Zolotarev (1986):

$$
\begin{aligned}
& c\left(\tau, H_{\tau}\right)=\exp (-i \tau) \int_{-d}^{\infty}\left(H_{0}+\gamma Z_{\tau}-S\right) f_{Y_{\tau}}\left(y_{\tau}, \alpha, \beta\right) \mathrm{d} Y_{\tau} \\
& c\left(\tau, H_{\tau}\right)=\exp (-i \tau)\left(\left(H_{0}-S\right) \int_{-d}^{\infty} f_{Y_{\tau}}\left(y_{\tau}, \alpha, \beta\right) \mathrm{d} Y_{\tau}+\gamma \int_{-d}^{\infty} Z_{\tau} f_{Y_{\tau}}\left(y_{\tau}, \alpha, \beta\right) \mathrm{d} Y_{\tau}\right) \\
& c\left(\tau, H_{\tau}\right)=\exp (-i \tau)\left(\left(H_{0}-S\right) \int_{-\infty}^{d} f_{Y_{\tau}}\left(y_{\tau}, \alpha,-\beta\right) \mathrm{d} Y_{\tau}+\gamma \int_{-\infty}^{d} Z_{\tau} f_{Y_{\tau}}\left(y_{\tau}, \alpha,-\beta\right) \mathrm{d} Y_{\tau}\right)
\end{aligned}
$$

Por lo tanto, la valuación de las opciones europeas de compra sobre sobre el índice de calentamiento diario con el modelo $\alpha$-estable es:

$$
\begin{aligned}
& c\left(\tau, H_{\tau}\right)=\exp (-i \tau)\left(\left(H_{0}-S\right) \Phi(d, \alpha,-\beta)+\gamma f_{Y_{\tau}}(d, \alpha,-\beta)\right) \\
& c\left(\tau, H_{\tau}\right)=\exp (-i \tau)\left(\left(H_{0}-S\right)(1-\Phi(-d, \alpha, \beta))+\gamma f_{Y_{\tau}}(-d, \alpha, \beta)\right)
\end{aligned}
$$

donde $d=\frac{H_{0}-S_{H}}{\gamma}$ y $H_{0}=\sum_{k=1}^{n} \operatorname{máx}\left(T_{0}-T_{k}, 0\right)$. 
La valuación de las opciones europeas de compra sobre sobre el índice de enfriamiento diario con el modelo $\alpha$-estable es:

$$
\begin{aligned}
& c\left(\tau, C_{\tau}\right)=\exp (-i \tau)\left(\left(C_{0}-S\right) \Phi(d, \alpha,-\beta)+\gamma f_{Y_{\tau}}(d, \alpha,-\beta)\right) \\
& c\left(\tau, C_{\tau}\right)=\exp (-i \tau)\left(\left(C_{0}-S\right)(1-\Phi(-d, \alpha, \beta))+\gamma f_{Y_{\tau}}(-d, \alpha, \beta)\right) \\
& \text { donde } d=\frac{C_{0}-S_{C}}{\gamma} \text { y } C_{0}=\sum_{k=1}^{n} \operatorname{máx}\left(T_{k}-T_{0}, 0\right) .
\end{aligned}
$$

Valuación de opciones de compra sobre los índices de temperatura

La valuación de opciones europeas de compra sobre los índices de temperatura es realizada con datos de 2018. Los resultados de los índices mensuales son presentados en la Tabla 10.

Tabla 10

Índices de temperatura de 2018

\begin{tabular}{ccc}
\hline 2018 & $H_{0}$ & $C_{0}$ \\
\hline Enero & 106.75 & 0.00 \\
Febrero & 76.75 & 1.45 \\
Marzo & 7.70 & 39.20 \\
Abril & 5.05 & 62.85 \\
Mayo & 0.95 & 118.55 \\
Junio & 0.95 & 94.30 \\
Julio & 6.70 & 32.70 \\
Agosto & 4.80 & 33.10 \\
Septiembre & 10.55 & 26.50 \\
Octubre & 28.85 & 22.50 \\
Noviembre & 73.90 & 1.75 \\
Diciembre & 123.54 & 0.00 \\
Total & 446.49 & 432.90 \\
\hline
\end{tabular}

Fuente: Elaboración propia con datos del observatorio central de Tacubaya

La Tabla 10 presenta los índices de grados de calentamiento y de enfriamiento diarios de 2018 y se observa asimetría negativa en el total de grados de calentamiento con respecto a los grados de enfriamiento, es decir, la demanda de grados de calentamiento anuales es $446.49^{\circ} \mathrm{C}$ y la demanda de grados de enfriamiento anuales es $432.90^{\circ} \mathrm{C}$. Los grados de calentamiento 
presentan una demanda estacional donde la demanda mínima se presenta en mayo y junio, aumentando de julio a diciembre, alcanzando un máximo en diciembre, y disminuyendo de enero a junio. Los grados de enfriamiento también presentan demanda estacional donde la demanda mínima se presenta en diciembre y enero, aumentando a partir de febrero, alcanzando un máximo en mayo, y disminuyendo de junio a noviembre. Los precios de liquidación propuestos suponiendo que la capacidad o necesidad de compra de grados de calentamiento y enfriamiento para 2019 son presentados en la Tabla 11.

Tabla 11

Precios de liquidación para 2019

\begin{tabular}{ccc}
\hline 2019 & $S_{H}$ & $S_{C}$ \\
\hline Enero & 100 & 1 \\
Febrero & 70 & 2 \\
Marzo & 15 & 35 \\
Abril & 8 & 60 \\
Mayo & 3 & 110 \\
Junio & 1 & 90 \\
Julio & 3 & 30 \\
Agosto & 8 & 30 \\
Septiembre & 15 & 25 \\
Octubre & 35 & 20 \\
Noviembre & 70 & 2 \\
Diciembre & 110 & 1 \\
Total & 438.00 & 406.00 \\
\hline
\end{tabular}

Fuente: Elaboración propia

La Tabla 11 presenta los precios de liquidación para 2019; son una propuesta en función de la información de los datos del observatorio de 1958 a 2018 y son un supuesto de la capacidad o necesidad de compra de grados de calentamiento o enfriamiento para 2019, planteado en un mercado extrabursátil donde los inversionistas con la posición larga buscan una cobertura de acuerdo a las necesidades de negocio para minimizar el costo de capital por la demanda de grados de calentamiento o enfriamiento en función de la temperatura de la ciudad de México. La valuación de las opciones europeas de compra sobre los índices calentamiento y enfriamiento son presentadas en la Figura 5. 


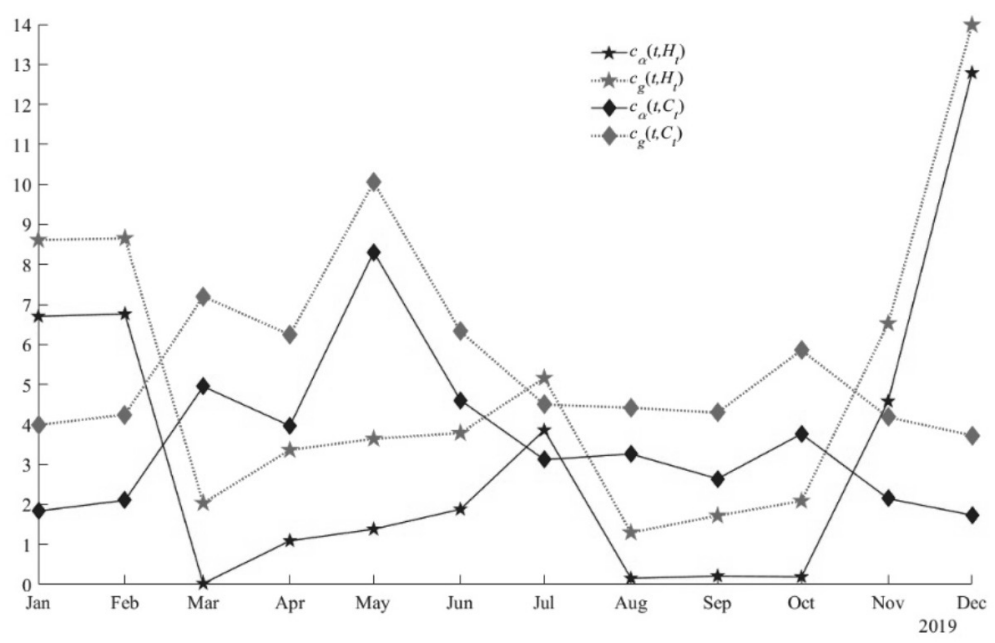

Figura 5. Valuación de opciones europeas de compra sobre los índices

Fuente: Elaboración propia

La Figura 5 presenta la valuación de las opciones europeas de compra $\alpha$-estables y gaussianas sobre los índices de calentamiento y enfriamiento mensual valuadas al iniciar el año 2019. Las opciones gaussianas son más caras que las opciones $\alpha$-estables, pero el objetivo no es comparar los modelos porque el modelo $\alpha$-estable es estadísticamente significativo. Las opciones gaussianas son más caras que las $\alpha$-estables y las coberturas anuales son:

$$
c_{\alpha}\left(t, C_{t}\right)=42.41, c_{g}\left(t, C_{t}\right)=65.01, c_{\alpha}\left(t, H_{t}\right)=39.60, c_{g}\left(t, H_{t}\right)=60.84
$$

donde la cobertura gaussiana anual sobre el índice de enfriamiento (65.01) es superior a la cobertura $\alpha$-estable (42.41) y la cobertura gaussiana anual sobre el índice de calentamiento (60.84) es superior a la $\alpha$-estable (39.60). La valuación de las opciones europeas de compra es realizada de forma mensual y la prima anual es calculada como el valor actual de las doce coberturas. En función del modelo estocástico de reversión a la media para 2019 son calculados los pagos de liquidación de las opciones y los resultados son presentados en la Tabla 12. 
Tabla 12

Pagos de liquidación de las opciones de compra

\begin{tabular}{ccc}
\hline 2019 & $H_{T}$ & $C_{T}$ \\
\hline Enero & 0.00 & 0.41 \\
Febrero & 0.00 & 12.60 \\
Marzo & 0.00 & 6.36 \\
Abril & 0.00 & 32.55 \\
Mayo & 0.00 & 0.00 \\
Junio & 0.00 & 0.00 \\
Julio & 0.00 & 27.02 \\
Agosto & 0.00 & 13.40 \\
Septiembre & 0.00 & 0.00 \\
Octubre & 0.00 & 5.15 \\
Noviembre & 0.00 & 0.00 \\
Diciembre & 0.00 & 0.00 \\
Total & 0.00 & 97.49 \\
\hline
\end{tabular}

Fuente: Elaboración propia

La Tabla 12 presenta los pagos de liquidación suponiendo, por simplicidad, que el costo por grado de calentamiento o enfriamiento es una unidad monetaria, los grados de calentamiento necesarios son menores que los grados de liquidación, entonces, las opciones de compra sobre el índice de calentamiento están fuera de dinero, en cambio, los grados de enfriamiento de enero a marzo, julio, agosto, octubre y diciembre son mayores que los grados de liquidación, recuperando la prima por la cobertura, entonces, la propuesta es que las temperaturas de referencia son estimadas estadísticamente en función de la ubicación, y las necesidades del producto o servicio y los costos que estas necesidades tienen para la estimación de los índices y las coberturas. En la ciudad de México son más necesarios los grados de enfriamiento (449.64) que los de calentamiento (267.39), por lo tanto, la temperatura de referencia es un parámetro en función de las necesidades de temperatura del producto o servicio y de la geolocalización.

Si la empresa toma la posición larga en el bono invertido a la tasa de interés libre de riesgo para cubrir los grados de enfriamiento diarios al final del año, $S_{C} \exp (-i T)$, para recibir $\$ 406$ y toma la posición larga en la opción de compra sobre los grados de enfriamiento, entonces, ahorra \$449.64 por costos del consumo energético para enfriar por una prima de $\$ 42.41 \mathrm{y}$, si la empresa toma la posición larga en el bono invertido a la tasa de interés libre de riesgo para cubrir los grados de calentamiento al final del año, $S_{H} \exp (-i T)$, para recibir $\$ 438$ y toma la posición larga en la opción de compra sobre los grados de calentamiento, entonces, 
tiene los $\$ 438$ por costos del consumo energético para calentar por una prima de $\$ 39.60$, la cual expira sin generar ahorros porque está fuera de dinero.

La importancia de tener información para la estimación de índices para valuar coberturas con productos derivados está de manifiesto.

\section{Conclusiones}

Las temperaturas presentan un comportamiento estacional, una tendencia positiva y significativa, los periodos más fríos se presentan entre octubre y marzo, y los periodos más cálidos se presentan entre marzo y junio. Las temperaturas analizadas presentan distribuciones leptocúrticas y con asimetrías negativas, entonces, las distribuciones $\alpha$-estables son pertinentes para modelar el proceso estocástico de reversión a la media para la valuación de opciones climáticas sobre los índices de temperatura. Los incrementos de las temperaturas promedio durante los años analizados son de $2.04^{\circ} \mathrm{C}$ y significativos.

El modelo determinista de reversión a la media es significativo e indica que la amplitud de la función de variación periódica es $2.35^{\circ} \mathrm{C}$, es decir, la diferencia entre temperaturas promedio es $4.7^{\circ} \mathrm{C}$, entonces, el modelo determinista de reversión a la media captura más adecuadamente que el modelo de regresión lineal simple el comportamiento estacional y las tendencias positivas, por lo tanto, el modelo determinista de reversión a la media es significativo para modelar las temperaturas pero es insuficiente para la valuación de las opciones de compra sobre los índices de temperatura y valuar la cobertura para la empresa.

El objetivo de la valuación de opciones climáticas sobre los índices de temperatura es alcanzado con la propuesta para aplicar una ecuación diferencial estocástica utilizando un proceso estocástico $\alpha$-estable y una solución que permite simular las temperaturas. Los parámetros $\alpha$-estables mensuales son estimados, y son significativos, concluyendo por medio de las pruebas de bondad de ajuste AD y CvM que la valuación de opciones sobre los índices de temperatura en mercados incompletos es pertinente con distribuciones $\alpha$-estables.

El estimador para el parámetro de reversión a la media es propuesto en función del parámetro de escala que permite simular variables aleatorias para pronosticar las temperaturas diarias de 2019; se indica la condición final para el precio de las opciones sobre los índices de temperatura, y un modelo para de valuación de opciones europeas de compra sobre los índices de temperatura es propuesto con distribuciones $\alpha$-estables, entonces, las opciones de compra sobre los índices son valuadas, y se concluye que las opciones $\alpha$-estables son pertinentes y estadísticamente significativas, en todos los casos tienen precios menores o iguales que las opciones gaussianas. Las opciones sobre los índices de temperatura representan una oportunidad para minimizar el costo derivado por el consumo energético para aumentar o disminuir la temperatura y un beneficio para las empresas y los consumidores. Las principales 
contribuciones de este trabajo son: aplicar una ecuación diferencial estocástica con procesos $\alpha$-estables justificando su pertinencia, estimar los parámetros de reversión a la media y resolver la ecuación diferencial estocástica para valuar opciones sobre los índices de temperatura con una ecuación cerrada que es una aplicación de procesos $\alpha$-estables estadísticamente significativos, y además la solución de la ecuación diferencial estocástica permite aplicar la simulación Monte Carlo para valuar opciones que no tienen soluciones cerradas aplicando procesos $\alpha$-estables y considerando efectos estacionales, cíclicos, calentamiento, reversión a la medía estadísticamente significativas.

El trabajo resalta la importancia de contar con información de las temperaturas a través de Internet e índices climáticos en función de la ubicación porque la temperatura es un factor de costos para las empresas y con las coberturas para la demanda de los índices de calentamiento o de enfriamiento necesarios es posible minimizar pérdidas económicas por el consumo energético.

En trabajos futuros es posible comparar el modelo con los precios de opciones sobre los grados de calentamiento o enfriamiento para los índices negociados en los mercados organizados o extrabursátiles mexicanos.

\section{Referencias}

Alaton, P., Djehiche, B. y Stillberger, D. (2002). On modelling and pricing weather derivatives, Applied Mathematical Finance, 9(1): 1-20. https://doi.org/10.1080/13504860210132897

Alva Vázquez, A. y Sierra Juárez, G. (2010). Opciones climáticas para el sector pesquero del pacífico mexicano, Panorama Económico, VI(11): 29-61. https://doi.org/10.29201/pe-ipn.v6i11.74

Benth, F. E. (2003). On arbitrage-free pricing of weather derivatives based on fractional brownian motion. Applied Mathematical Finance, 10(4): 303-324.

https://doi.org/10.1080/1350486032000174628

Benth F.E., Härdle W.K., López Cabrera B. (2011) Pricing of Asian temperature risk. Statistical Tools for Finance and Insurance. Springer, Berlin, Heidelberg.

https://doi.org/10.1007/978-3-642-18062-0_5

Black, F. y Scholes, M. (1973). The pricing of options and corporate liabilities, Journal of Political Economy, 81: 637-654. https://doi.org/10.1086/260062

Brody, D. C., Syroka, J. y Zervos, M. (2002). Dynamical pricing of weather derivatives. Quantitative Finance, 2(3): 189-198. https://doi.org/10.1088/1469-7688/2/3/302

Chang, S. y Tang, W. (2016). A fitted finite-volume method combined with the Lagrangian derivative for the eeather option pricing, Model Computational Methods in Applied Mathematics, 16(1): 17-33. https://doi.org/10.1515/ cmam-2015-0030

Considine, G. (2000). Introduction to weather derivatives, Weather Derivatives Group, Aquila Energy. www. agroinsurance.com/files/weather\%20derivatives.pdf (consultado el 01-10-2018).

Dzupire, N. C., Ngare, P., y Odongo, L. (2019). Pricing basket weather derivatives on rainfall and temperature processes, International Journal of Financial Studies, 7(3): 1-14.

https://doi.org/10.3390/ijfs7030035 
Goncu, A. (2011). Pricing temperature-based weather contracts: an application to China, Applied Economics Letters, 18: 1349-1354. https://doi.org/10.1080/13504851.2010.537619

Groll, A., López Cabrera, B. y Meyer Brandis, T. (2016). A consistent two-factor model for pricing temperature derivatives, Energy Economics, 55(2): 112-126.

https://doi.org/10.1016/j.eneco.2015.12.020

Huang, W., Yang, S. S., Chang, C. (2018). Modeling temperature behaviors: Application to weather derivative valuation, The Journal of Futures and Markets, 38(9): 1152-1175.

https://doi.org/10.1002/fut.21923

Jain, G. y Baile, C. (2000). Managing weather risks, Strategic Risk, September: 28-31.

Manfredo, M. R. y Richards, T. J. (2009). Hedging with weather derivatives: a role for options in reducing basis risk, Applied Financial Economics, 19: 87-97.

https://doi.org/10.1080/09603100701765166

Musshoff, O., Odening, M. y Xu, W. (2009). Management of climate risks in agriculture -will weather derivatives permeate?, Applied Economics, 43(9): 1-11.

https://doi.org/10.1080/00036840802600210

Nave Pineda, J. M. y González Sánchez, M. (2010). Valoración de derivados sobre el clima a partir de la modelización estocástica de la temperatura en el Aeropuerto Eldorado de Bogotá. Cuadernos de Administración, 23 : 261-283. https://doi.org/10.11144/Javeriana.cao23-41.vdsc

Platen, E. y West, J. (2004). A fair pricing approach to weather derivatives. Asian Pacific Financial

Markets, 11(1): 23-53. https://doi.org/10.1007/s10690-005-4252-9

Samorodnitsky, G. y Taqqu, M. (1994). Stable non-Gaussian random processes: Stochastic models with infinite variance. First edition, New York: Chapman and Hall.

https://doi.org/10.1201/9780203738818

Tang, W. y Chang, S. (2016). A Semi-Lagrangian method for the weather options of mean-reverting Brownian motion with jump-diffusion, Computers and Mathematics with Applications, 71(5): 1045-1058. https://doi. org/10.1016/j.camwa.2015.12.040

Uhlenbeck, G. E. y Ornstein, L. S. (1930). On the theory of Brownian motion. Physical Review, 36: 823-841. https://link.aps.org/doi/10.1103/PhysRev.36.823

Zolotarev, V. M. (1986). One-Dimensional stable distributions, AmericanMathematical Society. First edition. https://doi.org/10.1090/mmono/065 